\title{
SOLVABILITY OF THE FIRST COUSIN PROBLEM AND \\ VANISHING OF HIGHER COHOMOLOGY GROUPS FOR DOMAINS WHICH ARE NOT DOMAINS OF HOLOMORPHY ${ }^{1}$
}

\author{
BY AVNER FRIEDMAN
}

Communicated by F. Browder, June 7, 1965

This work is a sequel to [1]: In [1] we considered the vanishing of the first cohomology groups with coefficients in $\mathcal{O}, \mathcal{O}^{*}$ for sets $X \backslash A$ whereas in the present work we consider the same question for higher cohomology; at the same time we obtain some additional results for the first Cousin problem. As in [1] we take $n \geqq 3$.

Scheja [3] proved that if $X$ is an open set in $C^{n}$ and $A$ is an analytic closed subset of $X$ of dimension $\leqq n-q-2$, then the natural homomorphism

$$
H^{q}(X, \mathcal{O}) \rightarrow H^{q}(X \backslash A, \mathcal{O})
$$

is bijective. We shall prove:

THEOREM 1. Let $A$ be a closed bounded generalized polydisc in an open set $X$ of $C^{n}$. Then the natural homomorphism (1) is bijective for any $1 \leqq q \leqq n-2$.

Proof. Set $A=L_{1} \times \cdots \times L_{n}$ and let $K=K_{1} \times \cdots \times K_{n}$ be an open generalized polydisc with $A \subset K \subset \bar{K} \subset X$. Set $L^{\prime}=L_{2} \times \cdots$ $\times L_{n}, \quad K^{\prime}=K_{2} \times \cdots \times K_{n}, \quad G_{0}=\left(K_{1} \backslash L_{1}\right) \times K^{\prime}, \quad G_{1}=K_{1} \times\left(K^{\prime} \backslash L^{\prime}\right)$, $G=G_{0} \cup G_{1}$. By a straightforward generalization of [3, Hilfsatz] one gets $H^{q}(G, \mathcal{O})=0$. We now introduce a covering $U=\left\{U_{i}\right\}$ of $X \backslash A$ where all the $U_{i}$ are domains with $H^{q}\left(U_{i}, \mathcal{O}\right)=0$ and precisely $q+1$ of them, say $U_{i_{0}}, \cdots, U_{i_{q}}$, coincide with $G$. By Leray's theorem [2], the canonical homomorphism

$$
H^{q}(N(U), \mathcal{O}) \rightarrow H^{q}(X \backslash A, \mathcal{O})
$$

(where $N(U)$ is the nerve of $U$ ) is bijective.

We next introduce a covering $U^{\prime}=\left\{U_{i}^{\prime}\right\}$ of $X$ where $U_{i_{0}}^{\prime}=\cdots$ $=U_{i_{q}}^{\prime}=K_{1} \times K^{\prime}$ and $U_{i}^{\prime}=U_{i}$ for all other indices $i$. Again, the canonical map

$$
H^{q}\left(N\left(U^{\prime}\right), \mathcal{O}\right) \rightarrow H^{q}(X, \mathcal{O})
$$

1 This work was partially supported by the Alfred P. Sloan Foundation and by the NASA Grant NGR 14-007-021. 
is bijective. We shall now construct a map

$$
H^{q}(N(U), \mathcal{O}) \rightarrow H^{q}\left(N\left(U^{\prime}\right), \mathcal{O}\right) .
$$

Let $f \in H^{q}(N(U), \mathcal{O})$. We may view it as a $q$-cocycle. Let $f_{i_{0} \cdots i_{q}}$ be the section of $f$ on $U_{i_{0}} \cap \cdots \cap U_{i_{q}}=G$. The proof of Lemma 3 in [1] can be extended to show that $f_{i_{0} \cdots i_{q}}$ can be continued analytically to $K_{1} \times K^{\prime}$. The continued function $f_{i_{0}}^{\prime} \cdots i_{q}$ thus obtained is defined on $U_{i_{0}}^{\prime} \cap \cdots \cap U_{i_{q}}^{\prime}$. We now define $f_{j_{0}}^{\prime} \cdots j_{q}$ for any set of distinct indices $\left\{j_{0}, \cdots, j_{q}\right\}$ which does not coincide with the set $\left\{i_{0}, \cdots, i_{q}\right\}$. Since among the $j_{h}$ 's there is at least one index, say $i$, with $i \neq i_{k}$ for all $0 \leqq k \leqq q$, and, consequently, $U_{i}^{\prime}=U_{i} \subset X \backslash A$, we have $U_{i}^{\prime} \cap\left(K_{1} \times K^{\prime}\right)=U_{i}^{\prime} \cap G$. Hence $U_{j_{0}}^{\prime} \cap \cdots \cap U_{j_{q}}^{\prime}=U_{j_{0}} \cap \cdots \cap U_{j_{q}}$ and we can take $f_{j_{0}}^{\prime} \cdots j_{q}=f_{j_{0} \cdots j_{q}}$.

We have thus defined a $q$-cochain $f^{\prime}$ on $N\left(U^{\prime}\right) . f^{\prime}$ is cocycle. Indeed, observing that $U_{j_{0}}^{\prime} \cap \cdots \cap U_{j_{q+1}}^{\prime}$ coincides with $U_{j_{0}} \cap \cdots \cap U_{j_{q+1}}$ if all the $j_{k}$ are distinct from each other, and that the analytic function $f_{j_{0}}^{\prime} \cdots \hat{j}_{v} \cdots j_{q_{+1}}$ restricted to either of these sets coincides with $f_{j_{0}} \cdots y_{p} \cdots j_{q+1}$, the equation $\delta f=0$ implies $\delta f^{\prime}=0$.

We next show that if $f=\delta g$ then there is a $(q-1)$-chain $g^{\prime}$ with $\delta g^{\prime}=f^{\prime}$. If (a) $\left\{j_{0}, \cdots, j_{q-1}\right\} \subset\left\{i_{0}, \cdots, i_{q}\right\}$ then we take $g_{j_{0}}^{\prime} \cdots j_{q-1}$ to be the analytic continuation of $g_{j_{0} \cdots j_{q-1}}$ to $U_{j_{0}}^{\prime} \cap \cdots \cap U_{j_{q-1}}^{\prime}$, whereas if (a) does not hold then $U_{j_{0}}^{\prime} \cap \cdots \cap U_{j_{q-1}}^{\prime}$ $=U_{j_{0}} \cap \cdots \cap U_{j_{q-1}}$ and we take $g_{j_{0} \cdots j_{q-1}}^{\prime}=g_{j_{0} \cdots j_{q-1}}$. With $g^{\prime}$ thus constructed, the relation $\delta g^{\prime}=f^{\prime}$ over $U_{j_{0}}^{\prime} \cap \cdots \cap U_{j_{q}}^{\prime}$ in case (b) $\left\{j_{0}, \cdots, j_{q}\right\}=\left\{i_{0}, \cdots, i_{q}\right\}$ follows from the relation $\delta g=f$ over $U_{j_{0}} \cap \cdots \cap U_{j_{q}}$ by analytic continuation, whereas in case (b) does not hold it coincides with the relation $\delta g=f$ over $U_{j_{0}} \cap \cdots \cap U_{j_{q}}$.

We have thus shown that the map $f \rightarrow f^{\prime}$ defines a homomorphism (4). This map is surjective since, given $f^{\prime}$, its restriction $f$ to $N(U)$ is mapped into $f^{\prime}$ by the above map. It is also injective since if $f^{\prime}=\delta g^{\prime}$ for some $(q-1)$-cochain $g^{\prime}$ over $N\left(U^{\prime}\right)$, then the restriction $g$ of $g^{\prime}$ to $N(U)$ clearly satisfies $f=\delta g$. Noting finally that the map $f \rightarrow f^{\prime}$ is the inverse of the restriction map, and combining (2)-(4), (1) follows.

Corollary. If $H^{q}(X, \mathcal{\theta})=0$ then $H^{q}(X \backslash A, \mathcal{\theta})=0$. In particular, if $X$ is Cousin I then $X \backslash A$ is Cousin I.

THEOREM 2. Let $A, B$ be two closed bounded subsets of an open set $X \subset C^{n}$ and let $P$ be a closed generalized polydisc with $A \subset$ int $P \subset P$ $C$ int $B$. If, for some $1 \leqq q \leqq n-2$, the natural homomorphism

$$
H^{q}(X \backslash A, \mathcal{O}) \rightarrow H^{q}(X \backslash B, \mathcal{O})
$$

is injection, then there exists a homomorphism $\lambda: H^{q}(X \backslash A, \mathcal{O}) \rightarrow H^{q}(X, \mathcal{O})$ 
such that $\pi \lambda=i d e n t i t y$, where $\pi$ is the map (1) (and, consequently, $\pi$ is surjective); in particular, if $H^{q}(X, \mathcal{O})=0$ then $H^{q}(X \backslash A, \mathcal{O})=0$.

Proof. Take coverings $U^{1}, U^{2}, U^{3}, U^{4}$ of $X, X \backslash A, X \backslash P, X \backslash B$ respectively whose open sets are domains of holomorphy and such that the sets of $U^{i}(i=2,3,4)$ are among the sets of $U^{i-1}$. Given $f_{2}$ $\in H^{q}\left(N\left(U^{2}\right), \theta\right)$ there corresponds to it (by restriction) a unique element $f_{4}$ in $H^{q}\left(N\left(U^{4}\right), \theta\right)$ and a unique element $f_{3}$ in $H^{q}\left(N\left(U^{3}\right), \theta\right)$; $f_{4}$ is the restriction of $f_{3}$. By Theorem 1 there exists an $f_{1} \in H^{q}\left(N\left(U^{1}\right), \mathcal{O}\right)$ whose restriction to $N\left(U^{3}\right)$ is $f_{3}$. Hence the restriction of $f_{1}$ to $N\left(U_{4}\right)$ is $f_{4}$. Since $f_{1}$ and $f_{2}$ have the same restriction on $N\left(U^{4}\right)$, the injectivity of (5) implies that the restriction of $f_{1}$ to $N\left(U^{2}\right)$ is $f_{2}$. Thus the map $f_{2} \rightarrow f_{1}$ is an inverse of the restriction map $H^{q}\left(N\left(U^{1}\right), \theta\right) \rightarrow H^{q}\left(N\left(U^{2}\right), \mathcal{\theta}\right)$. The assertion of the theorem now follows with $\lambda$ being the image of the homomorphism $f_{2} \rightarrow f_{1}$ under the canonical map corresponding to $H^{q}\left(N\left(U^{2}\right), \theta\right) \rightarrow H^{q}(X \backslash A, \mathcal{\theta}), H^{q}\left(N\left(U^{1}\right), \mathcal{\theta}\right) \rightarrow H^{q}(X, \mathcal{\theta})$.

Generalizations. By successive applications of Theorem 1 we get:

(1) If $A_{1}, \cdots, A_{m}$ are closed bounded generalized polydiscs such that $A_{j} \cap A_{k}=\varnothing$ if $j \neq k$, then the natural map

$$
H^{q}(X, \mathcal{\theta}) \rightarrow H^{q}\left(X \backslash\left(\bigcup_{i=1}^{m} A_{i}\right), \mathcal{\theta}\right)
$$

is bijective.

(2) Theorem 1 extends to the case where $X$ is an open set on a complex manifold provided $A$ is contained in one coordinate patch and its image in $\boldsymbol{C}^{n}$ is a generalized polydisc. Theorem 2 and (1) have similar extensions.

By slightly modifying the proof of Theorem 1 we obtain:

(3) If $X=X_{1} \times K_{p+1} \times \cdots \times K_{n}, A=A_{1} \times K_{p+1} \times \cdots \times K_{n}$ where $X_{1}$ is any open set of $C^{p}$ and $K_{j}$ is an open set in the $z_{j}$-plane, then the homomorphism (1) is bijective if $1 \leqq q \leqq p-2$.

(4) If $A$ in Theorem 1 is convex, then (see [1]) $H^{q}\left(G, 0^{*}\right)=0$. By modifying the proof of Theorem 1 we find that the natural homomorphism

$$
H^{q}\left(X, \mathcal{O}^{*}\right) \rightarrow H^{q}\left(X \backslash A, \mathcal{O}^{*}\right)
$$

is bijective. The analogs of Theorem 2 and (1)-(3) are also valid.

We shall now give a different approach to proving results similar to Theorem 1. Since this approach does not yield a result as general as Theorem 1 , we shall only sketch it. Let $X=K_{1} \times \cdots \times K_{n}$, $A=L_{1} \times \cdots \times L_{n}$ be generalized polydiscs. We say that the condition $\left(\mathrm{A}_{m}\right)$ holds if for each $j=1, \cdots, m$ either (a) $K_{1}$ is the whole 
plane $C$ and then $L_{j}$ is an arbitrary closed bounded subset of $K_{j}$, or (b) $K_{j}=C$ and then $L_{j}$ consists of a finite number of points. The $L_{j}$ for $j=m+1, \cdots, n$ are arbitrary closed subsets of $K_{j}$.

THEOREM 3. If $\left(\mathrm{A}_{m}\right)$ holds for some $2 \leqq m \leqq n$ then $H^{q}(X \backslash A, \theta)=0$ for $1 \leqq q \leqq \min (m-1, n-2)$. The relations $H^{n-1}(X \backslash A, \theta) \neq 0$, $H^{q}(X \backslash A, \Theta)=0$ for $q \geqq n$ are valid under the assumption $\left(A_{0}\right)$.

Proof. Setting $\Delta_{j}=K_{1} \times \cdots \times K_{j-1} \times\left(K_{j} \backslash L_{j}\right) \times K_{j+1} \times \cdots \times K_{n}$ and noting that $H^{q}\left(\Delta_{j}, \mathcal{O}\right)=0$ for $q \geqq 1$, it suffices to consider $H^{q}((U), \mathcal{O})$, where $U=\left\{\Delta_{1}, \cdots, \Delta_{n}\right\}$. We consider only the case $1 \leqq q \leqq n-2$. Denote by $I_{j_{1} \cdots j_{k}}(h)$ the Cauchy integral of $h$ with the $i$ th contour being $\partial K_{i}$ if $i \neq j_{p}$ for all $p$, and $\partial L_{i}$ if $i=j_{p}$ for some $p$. (Actually one should replace $\partial K_{m}, \partial L_{m}$ by smooth $\partial K_{m, e}, \partial L_{m, e}$ which approximate $\partial K_{m}, \partial L_{m}$.) Then we can represent each component $f_{i_{0} \cdots i_{q}}$ of a $q$ cochain $f$ by

$$
f_{i_{0} \cdots i_{q}}=\sum_{k=0}^{q+1} \sum_{0 ; j_{1}<\cdots<j_{k}}^{q} I_{i_{1}} \cdots i_{j_{k}}\left(f_{i_{0}} \cdots i_{q}\right) .
$$

Lemma 1. Consider a domain $D=K \backslash L$ in the complex plane, where $K$ is the whole plane and $L$ is any closed bounded set with $C^{1}$ boundary $\partial L$. Let $\phi(z)$ be any analytic function in $D$ and let $\psi(z)$ be any continuous function on $\partial L$ such that

$$
\int_{|\zeta|-R} \frac{\phi(\zeta)}{\zeta-z} d \zeta+\int_{\partial L} \frac{\psi(\zeta)}{\zeta-z} d \zeta=0 \text { in } D \cap\{z ;|z|<R\}
$$

for all $R$ sufficiently large. Then, for all $R$ sufficiently large,

$$
\int_{|\zeta|=R} \frac{\phi(\zeta)}{\zeta-z} d \zeta=\int_{\partial L} \frac{\psi(\zeta)}{\psi-z} d \zeta=0 \text { in } D \cap\{z ;|z|<R\} .
$$

A similar result holds in case $K$ is a bounded set with $C^{1}$ boundary and $L$ consists of a finite number of points. Using these results, the condition $\delta f=0$ implies the following system of equations:

If $i_{0}<\cdots<i_{h} \leqq m<i_{h+1}<\cdots<i_{q+1}$ for some $0 \leqq h \leqq q+1$, and if $i_{j_{1}}<\cdots<i_{j_{k}} \leqq m$ for some $0 \leqq k \leqq h$, then

(7) $\sum_{p=0}^{q-h+1} \sum_{h+1 ; \lambda_{1}<\cdots<\lambda_{p}}^{q+1} I_{i j_{1}} \cdots i_{\left.j|j|\right|_{k} \lambda_{1} \cdots \lambda_{p}}\left(\sum_{\nu=0}^{q+1}(-1)^{\nu} f_{i_{0}} \ldots i_{p} \ldots i_{q}\right)=0$,

where in the third summation $\nu \neq j_{1}, \cdots, \nu \neq j_{k}$ and $\nu \neq \lambda_{1}, \cdots, \nu \neq \lambda_{p}$.

To find $g$ satisfying $\delta g=f$, we try to represent $g_{i_{0}} \ldots i_{g-1}$ analogously to (6), and then the relation $\delta g=f$ is a consequence of the following system of equations: 
If $i_{0}<\cdots<i_{h-1} \leqq m<i_{h}<\cdots<i_{q}$ for some $0 \leqq h-1 \leqq q$, and if $i_{j_{1}}<\cdots<i_{j_{k}} \leqq m$ for some $0 \leqq k \leqq h-1$, then

$$
\begin{aligned}
& \sum_{p=0}^{q-h+1} \sum_{h ; \lambda_{1}<\cdots<\lambda_{p}}^{q} I_{i j_{1} \ldots i j_{k} \lambda_{1}} \ldots i \lambda_{p}\left(\sum_{\nu=0}^{q}(-1)^{\nu} g_{i_{0}} \ldots \hat{i}_{\nu} \ldots i_{q}\right) \\
& -\sum_{p=0}^{q-h+1} \sum_{h ; \lambda_{1}<\cdots<\lambda_{p}}^{q} I_{i j_{1} \cdots i j_{k} i \lambda_{1} \cdots i \lambda_{p}}\left(f_{i_{0} \cdots i_{q}}\right)=0,
\end{aligned}
$$

where in the third summation of the first term $\nu \neq j_{1}, \cdots, \nu \neq j_{k}$ and $\nu \neq \lambda_{1}, \cdots, \nu=\lambda_{p}$.

Using (7) we can solve (8) as follows: If $i_{0}>1$, or if $i_{0}=1, i_{j_{1}}>1$ then $g_{i_{0} \cdots i_{q-1}}=f_{i_{0} \cdots i_{q-1}}$. If $i_{0}=i_{j_{1}}=1$ and if $i_{1}>2$ or $i_{1}=2, i_{j_{2}}>2$ then $g_{i_{0} \cdots i_{q-1}}=f_{2 i_{0} \cdots i_{q-1}}$. We proceed in this manner and finally define, in case $i_{0}=i_{j_{1}}=1, \cdots, i_{k-1}=i_{j_{k}}=k, g_{i_{0} \cdots i_{q-1}}=f_{k+1, i_{0} \cdots i_{q-1}}$.

This method extends also to the situations described in (1), (3) above.

Added in proof. The relation $H^{p-2}(X \backslash A, \theta) \neq 0$ holds if in (3) $X_{1}$ and $A_{1}$ are both generalized polydiscs. Taking $\Omega_{m}=X_{m} \backslash A_{m}$ where $X_{m}, A_{m}$ are generalized polydiscs with $X_{m} \searrow \bar{X}, A_{m} \nearrow A$ one derives, for fixed $1 \leqq q \leqq n-2$, examples of domains $\Omega_{m}$ with $\Omega_{m-1} \supset \bar{\Omega}_{m}$, such that $H^{r}\left(\Omega_{m}, \mathcal{\theta}\right)=0$ for $1 \leqq r \leqq n-2$ but $H^{q}(\Omega, \mathcal{\theta}) \neq 0$ where $\Omega=\operatorname{int}\left(\lim \Omega_{m}\right)$.

By Dolbeault's theorem, $H^{q}(\Omega, \mathcal{\theta})=0$ if and only if for any $C^{\infty}(\Omega)$ form $f$ of bidegree $(0, q)$ with $\bar{\partial} f=0$ there is a $C^{\infty}(\Omega)$ form $u$ with $\bar{\partial} u=f$. By modifying the proof in $[2$, p. 29] we find: If for some $q>1, \bar{\Omega}_{m} \subset \Omega_{m+1}, \Omega=\lim \Omega_{m}, H^{r}\left(\Omega_{m}, \mathcal{\theta}\right)=0$ for $r=q-1, q$, then $H^{q}(\Omega, \mathcal{\theta})=0$. Also if $H^{1}\left(\Omega_{m}, \mathcal{\theta}\right)=0$ and if for any $u$ holomorphic in $\Omega_{m}$ and $\epsilon>0$ there is a $v$ holomorphic in $\Omega_{m+1}$ with $|u-v|<\epsilon$ in $\Omega_{m-1}$, then $H^{1}(\Omega, \vartheta)=0$; this can be applied to $\Omega_{m}=X_{m} \backslash A_{m}$ as in $[1$, Theorem 3].

\section{REFERENCES}

1. A. Friedman, On the Cousin problems, Bull. Amer. Math. Soc. 71 (1965), 737741.

2. R. C. Gunning and H. Rossi, Analytic functions of several complex variables, Prentice-Hall, 1965.

3. G. Scheja, Riemannsche Helbarkeitssätze für Cohomologieklassen, Math. Ann. 144 (1961), 345-360.

NORTHWESTERN UNIVERSITY 